\title{
SÍNTESE E AVALIAÇÃO DA ATIVIDADE FITOTÓXICA DE NOVOS ANÁLOGOS OXIGENADOS DO ÁCIDO HELMINTOSPÓRICO
}

Luiz Cláudio de Almeida Barbosa*, Antônio Jacinto Demuner, Célia Regina Álvares Maltha e Patrícia Silvana da Silva Departamento de Química, Universidade Federal de Viçosa, 36571-000 Viçosa - MG

Antônio Alberto da Silva

Departamento de Fitotecnia, Universidade Federal de Viçosa, 36571-000 Viçosa - MG

Recebido em 24/7/02; aceito em 21/2/03

SYNTHESIS AND PHYTOTOXIC ACTIVITY EVALUATION OF NEW OXYGENATED ANALOGUES OF HELMINTHOSPORIC ACID. Several compounds related to helminthosporic acid (3) were synthesized via the [3+4] cycloaddition. The reaction of 3-hydroxymethyl-2-methylfuran (12) with 1,1,3,3-tetrabromo-4-methylpentan-2-one (13) resulted in 7hydroxymethyl-4 $\alpha$-isopropyl-1 $\alpha$-methyl-8-oxabicyclo[3.2.1] oct-6-en-3-one (8) (37\%) and 7-hydroxymethyl-2 $\alpha$-isopropyl-1 $\alpha$ methyl-8-oxabicyclo[3.2.1] oct-6-en-3-one (14) (12\%), which were converted into 7 -formyl-4 $\alpha$-isopropyl-1 $\alpha$-methyl-8oxabicyclo[3.2.1] oct-6-en-3-one (16) (32\% from 8) and 7-formyl-2 $\alpha$-isopropyl-1 $\alpha$-methyl-8-oxabicyclo[3.2.1]oct-6-en-3-one (18) (40\% from 14), respectively. Reduction of (8) resulted in 7-hydroxymethyl-4 $\alpha$-isopropyl-1 $\alpha$-methyl-8-oxabicyclo[3.2.1]oct-6en-3 $\alpha$-ol (11) (63\% from 8) and 7-hydroxymethyl-4 $\alpha$-isopropyl-1 $\alpha$-methyl-8-oxabicyclo[3.2.1]oct-6-en-3 $\beta$-ol (15) (30\% from 8). The $4 \alpha$-isopropyl-1 $\alpha$-methyl-3-oxo-8-oxabicyclo[3.2.1] oct-6-en-7-oic acid (19) was obtained by oxidation of (16) (78\%). The results of biological tests are described in details. The best result was observed for compound (15) that caused $76 \%$ inhibition on the root growth of D. tortuosum.

Keywords: [3+4] cycloaddition; helminthosporic acid; herbicides.

\section{INTRODUÇÃO}

Micoorganismos produzem uma grande variedade de compostos fitotóxicos com potencial para serem usados diretamente como herbicidas, ou como protótipos para a descoberta de novos herbicidas sintéticos. Ao contrário, as plantas não são referência para a obtenção de fitotoxinas e destacam-se como fonte de outros pesticidas, particularmente os inseticidas ${ }^{1,2}$.

Helmintosporol (1), helmintosporal (2) e ácido helmintospórico (3) são toxinas sesquiterpênicas naturais isoladas em culturas do fungo Helminthosporium sativum, um ascomiceto patogênico responsável pela ferrugem e apodrecimento da raiz de cereais ${ }^{3,4}$.
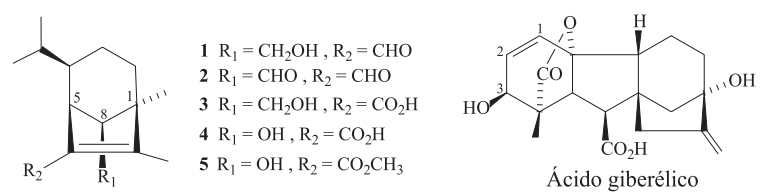

Testes biológicos realizados com o helmintosporol (1) incentivaram a investigação do potencial regulador do crescimento de plantas dessa classe de compostos, pois se observou rápido crescimento de brotos em plântulas de arroz e efeito inibitório sobre o crescimento de plântulas de trigo ${ }^{5,6}$.

$\mathrm{O}$ efeito regulador sobre o crescimento de plantas foi também constatado em derivados helmintospóricos com diferentes substituintes $R_{1}$ e $R_{2}$, sendo observadas, para os mesmos, respostas bastante distintas nos ensaios realizados com arroz e alface ${ }^{6}$. Há evidências de que a atividade biológica do helmintosporol (1), bem como do ácido helmintospórico (3), resulte da similaridade estrutural com dois dos anéis do ácido giberélico, um regulador do crescimento de plantas $^{7,8}$. A presença do grupo $3 \beta$-hidroxi na estrutura do ácido giberélico, unidade funcional importante para a existência da atividade biológica ${ }^{9}$, despertou o interesse para o estudo do potencial biológico de derivados do ácido helmintospórico, uma vez que há em sua estrutura o grupo hidroximetil em C8. Para avaliar a influência do grupo hidroxila em C8 sobre a atividade biológica, foram preparados ${ }^{7}$ os derivados (4) e (5), sendo observada para (4) atividade superior à do ácido helmintospórico (3). Por outro lado, observou-se que a atividade biológica foi extinta para o composto (5), éster metílico de (4).

O sucesso de herbicidas comerciais, cujos princípios ativos são de origem microbiana, desperta cada vez mais o interesse para a busca de compostos com efeito herbicida análogos a compostos oriundos de microorganismos. Recentemente relatamos a atividade biológica do 8-oxabiciclo[3.2.1] oct-6-en-3-ona (6) ${ }^{10}$ e dos derivados (7) $(\mathbf{1 1})^{11}$, análogos oxigenados do ácido helmintospórico, cujos resultados evidenciaram a potencialidade fitotóxica dessa classe de compostos. A presença do grupo isopropil parece ser um dos fatores determinantes para a atividade biológica, pois no ensaio realizado com o biciclo (6) não se observou efeito sobre a germinação e desenvolvimento em sorgo. Contrariamente, os compostos (7) - (11), na concentração de $100 \mu \mathrm{g} \mathrm{mL}{ }^{-1}$, promoveram estímulo ao crescimento radicular em pepino e inibição radicular em sorgo.

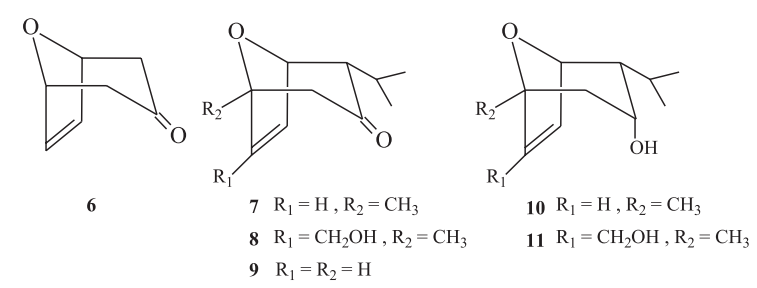


Neste trabalho descrevemos a síntese e avaliação da atividade fitotóxica de análogos oxigenados do ácido helmintospórico derivados do 8-oxabiciclo[3.2.1]oct-6-en-3-ona (6) (Esquema 1), que foram obtidos através da reação de cicloadição $[3+4]^{12}$. Os ensaios para avaliação da atividade fitotóxica dos compostos sintetizados foram realizados utilizando-se sorgo (Sorghum bicolor L.), pepino (Cucumis sativus L.), capim-braquiária (Brachiaria decumbens Stapf) e desmódio (Desmodium tortuosum (Sw.) DC.).

\section{PARTE EXPERIMENTAL}

\section{Procedimentos experimentais gerais}

As temperaturas de fusão foram determinadas em aparelho Kofler $\mathrm{R}$ Winkel Sotting. Os espectros na região do infravermelho foram registrados em pastilhas de $\mathrm{KBr}$ ou em solução de clorofórmio, em espectrômetro Perkin Elmer FTIR 1000, na região de 4000 a $600 \mathrm{~cm}^{-1}$. Os espectros de RMN ${ }^{1} \mathrm{H}$ e RMN ${ }^{13} \mathrm{C}$ foram obtidos em espectrômetro Bruker DPX200. Os deslocamentos químicos estão apresentados em partes por milhão $(\delta)$ relativos ao tetrametilsilano (TMS, $\delta=0,0$ ), e como solvente utilizou-se $\mathrm{CDCl}_{3}$. Os espectros de massas foram obtidos em espectrômetro Shimadzu QP5000. As condições estabelecidas foram: temperatura do injetor $\left(250^{\circ} \mathrm{C}\right)$, temperatura do detetor FID $\left(300{ }^{\circ} \mathrm{C}\right)$, temperatura programada para a coluna (de 60 a $200{ }^{\circ} \mathrm{C}$, com variação de $\left.5{ }^{\circ} \mathrm{C} \mathrm{min}^{-1}\right)$, coluna $\mathrm{BP} 1(30 \mathrm{~m} \times 0,22 \mathrm{~mm} \times 0,25 \mu \mathrm{m})$.

Os reagentes utilizados nas reações e os solventes utilizados em cromatografia em coluna de sílica gel foram previamente purificados e secados de acordo com os procedimentos descritos na literatu$\mathrm{ra}^{13}$. Para a purificação dos compostos utilizou-se a cromatografia em coluna de sílica gel 60 (70-230 mesh-ASTM, Merck). Todas as reações foram monitoradas por cromatografia em camada delgada utilizando-se placas de sílica gel Camlab-Polygram SILK/UV ${ }_{254}$, com $0,25 \mathrm{~mm}$ de espessura.

Os compostos (12) e (13) foram preparados de acordo com a metodologia descrita na literatura ${ }^{11}$.

\section{Procedimentos sintéticos}

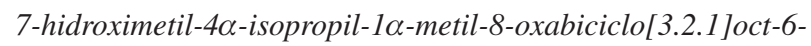
en-3-ona (8) e 7-hidroximetil-2 $\alpha$-isopropil-1 $\alpha$-metil-8oxabiciclo[3.2.1] oct-6-en-3-ona (14): A uma solução de 2hidroximetil-2-metilfurano (12) (1,0 g, 8,92 mmol) e 1,1,3,3tetrabromo-4-metilpentan-2-ona (13) $(2,0 \mathrm{~mL}, 10,0 \mathrm{mmol})$, em benzeno seco $(120 \mathrm{~mL})$, sob atmosfera de nitrogênio, agitação magnética e a $0{ }^{\circ} \mathrm{C}$, foi adicionado dietilzinco $(25 \mathrm{~mL}$ de uma solução 1,0 mol L ${ }^{-1}$, em hexano, 25,0 mmol) durante 1,5 h. A mistura foi mantida sob agitação magnética, à temperatura ambiente por $10 \mathrm{~h}$. Após decorrido esse tempo, adicionaram-se $100 \mathrm{~mL}$ de solução saturada de $\mathrm{NH}_{4} \mathrm{Cl}$. A mistura foi filtrada sob Celite e o produto foi extraído com diclorometano $(3 \times 30 \mathrm{~mL})$. A fase orgânica foi lavada com $40 \mathrm{~mL}$ de solução saturada de $\mathrm{NaCl}$, seca sobre $\mathrm{MgSO}_{4}$ e concentrada em evaporador rotatório. Foi obtido um óleo escuro (3,0 g, cicloadutos dibromados) que foi dissolvido em $15 \mathrm{~mL}$ de metanol. Em seguida, adicionou-se solução metanólica saturada de $\mathrm{NH}_{4} \mathrm{Cl}(30 \mathrm{~mL}$ de metanol e 3,5 g de $\mathrm{NH}_{4} \mathrm{Cl}$ ) e 8,0 g de amálgama de $\mathrm{Zn} / \mathrm{Cu}$. A mistura foi mantida sob agitação magnética, à temperatura ambiente durante $6 \mathrm{~h}$ e, então, filtrada sob Celite. Ao filtrado foram adicionados 45 $\mathrm{mL}$ de solução saturada de $\mathrm{Na}_{2}$ EDTA, $60 \mathrm{~mL}$ de diclorometano e logo após foi feita nova filtração sob Celite. A fase aquosa foi separada da fase orgânica e extraída com diclorometano $(3$ x $30 \mathrm{~mL})$. A fase orgânica resultante foi secada com $\mathrm{MgSO}_{4}$ e concentrada em evaporador rotatório. Foi obtido um óleo escuro, cuja purificação por cromatografia em coluna de sílica gel (hexano:éter dietílico 1:1) forneceu o álcool (8) (óleo amarelo, 0,677 g, 3,22 mmol, 37\%) e seu isômero (14) (óleo amarelo, 0,225 g, 1,07 mmol, 12\%).

Dados de (8): IV (filme líquido, $\mathrm{cm}^{-1}$ ) $\overline{\mathrm{v}}_{\max }: 3440,2961,2872$, 1707, 1465, 1380, 1326, 1196, 1164, 1041, 1010, 963, 941, 874, 852, 796. RMN ${ }^{1} \mathbf{H}\left(200 \mathrm{MHz}, \mathrm{CDCl}_{3}\right) \delta: 0,90\left(\mathrm{~d}, 3 \mathrm{H}, J_{10.9}=6,8 \mathrm{~Hz}\right.$, Me-10), 1,05 (d, 3H, $\left.J_{11,9}=6,8 \mathrm{~Hz}, \mathrm{Me}-11\right), 1,45$ (s, 3H, Me-12), 2,00 (octeto, $1 \mathrm{H}, J_{9,10}=J_{9,11}=J_{9,4}=7,3 \mathrm{~Hz}, \mathrm{H}-9$ ), $2,28(\mathrm{~s}, 1 \mathrm{H}, \mathrm{OH}$ ), $2,42\left(\mathrm{~d}, 1 \mathrm{H}, J_{2 \alpha, 2 \beta}=14,8 \mathrm{~Hz}, \mathrm{H}-2 \alpha\right), 2,53\left(\mathrm{~d}, 1 \mathrm{H}, J_{2 \beta, 2 \alpha}=14,8 \mathrm{~Hz}, \mathrm{H}-\right.$ $2 \beta), 2,54$ (dd, $\left.1 \mathrm{H}, J_{4,9}=7,3 \mathrm{~Hz}, J_{4,5}=3,0 \mathrm{~Hz}, \mathrm{H}-4\right), 4,13$ (d, $1 \mathrm{H}, J_{13 \mathrm{~b}, 13 \mathrm{a}}=$ $14,9 \mathrm{~Hz}, \mathrm{H}-13 \mathrm{~b}), 4,28$ (d, $\left.1 \mathrm{H}, J_{13 \mathrm{a}, 13 \mathrm{~b}}=14,9 \mathrm{~Hz}, \mathrm{H}-13 \mathrm{a}\right), 4,98$ (dd, $\left.1 \mathrm{H}, J_{5,4}=3,0 \mathrm{~Hz}, J_{5,6}=1,5 \mathrm{~Hz}, \mathrm{H}-5\right), 6,02\left(\mathrm{~d}, 1 \mathrm{H}, J_{6,5}=1,5 \mathrm{~Hz}, \mathrm{H}-6\right)$. RMN ${ }^{13} \mathbf{C}\left(\mathbf{5 0} \mathbf{M H z}, \mathbf{C D C l}_{3}\right) \delta: 19,9 *(\mathrm{Me}-10), 21,5^{*}(\mathrm{Me}-11), 22,5^{*}$ (Me-9), 24,5* (Me-12), 52,3 (C-2), 58,1 (C-13), 61,2 (C-4), 78,6 (C-5), 86,2 (C-1), 126,6 (C-6), 149,1 (C-7), 207,5 (C-3). EM, m/z (\%): $210\left(\mathrm{M}^{+}, \mathrm{C}_{12} \mathrm{H}_{18} \mathrm{O}_{3}, 3\right), 192$ (6), 111 (65), 107 (8), 83 (13), 69 (46), 55 (16), 43 (100).

Dados de (14): IV (filme líquido, $\mathrm{cm}^{-1}$ ) $\overline{\mathrm{v}}_{\max }: 3430,2961,2874$, 1704, 1668, 1464, 1381, 1198, 1162, 1041, 933, 867. RMN ${ }^{\mathbf{1}} \mathbf{H}(200$ $\left.\mathrm{MHz}_{2} \mathrm{CDCl}_{3}\right) \delta: 0,90\left(\mathrm{~d}, 3 \mathrm{H}, J_{10.9}=6,8 \mathrm{~Hz}, \mathrm{Me}-10\right), 1,19(\mathrm{~d}, 3 \mathrm{H}$, $J_{11,9}=6,8 \mathrm{~Hz}, \mathrm{Me}-11$ ), 1,53 (s, 3H, Me-12), 1,90 (m, 1H, H-9), 2,23 (d, $\left.1 \mathrm{H}, J_{4 \alpha, 4 \beta}=18,0 \mathrm{~Hz}, \mathrm{H}-4 \alpha\right), 2,49$ (m, $2 \mathrm{H}, \mathrm{H}-2$ e OH), 2,78 (dd, $1 \mathrm{H}, J_{4 \beta, 4 \alpha}=18,0 \mathrm{~Hz}$ e $\left.J_{4 \beta, 5}=5,9 \mathrm{~Hz}, \mathrm{H}-4 \beta\right), 4,35$ (sl, 2H, H-13a e H13b), 4,92 (d, 1H, $\left.J_{5,4 \beta}=5,9 \mathrm{~Hz}, \mathrm{H}-5\right), 6,24$ (m, 1H, H-6). RMN ${ }^{13} \mathbf{C}$ (50 MHz, CDCl $)$ ) $\delta: 19,4 * *(\mathrm{Me}-10), 21,5 * *(\mathrm{C}-11), 25,1 *(\mathrm{C}-9)$, 26,4* (C-12), 44,6 (C-2), 59,7 (C-13), 68,4 (C-4), 72,1 (C-5), 86,2 (C-1), 125,9 (C-6), 149,4 (C-7), 207,8 (C-3). *As atribuições podem estar trocadas.

7-hidroximetil-4 $\alpha$-isopropil-1 $\alpha$-metil-8-oxabiciclo[3.2.1]oct-6en- $3 \alpha$-ol (11) e 7-hidroximetil-4 $\alpha$-isopropil-1 $\alpha$-metil-8oxabiciclo[3.2.1] oct-6-en-3 $\beta$-ol (15): A uma solução do cicloaduto (8) $(0,09 \mathrm{~g}, 0,43 \mathrm{mmol})$ em etanol $(10 \mathrm{~mL})$, foi adicionado boroidreto de sódio $(0,05 \mathrm{~g}, 1,3 \mathrm{mmol})$. A mistura foi mantida sob aquecimento $\left(55^{\circ} \mathrm{C}\right)$ e agitação magnética durante $4 \mathrm{~h}$, sendo então resfriada em banho de gelo. Em seguida, foram adicionados $10 \mathrm{~mL}$ de água gelada e feita a extração com diclorometano $(3 \times 10 \mathrm{~mL})$. A fase orgânica foi secada com $\mathrm{MgSO}_{4}$ e concentrada em evaporador rotatório. Foi obtido um óleo amarelo que, após purificação por cromatografia em coluna de sílica (hexano:éter dietílico 1:5) forneceu o diol endo (11) (cristais brancos, 0,058 g, 0,27 mmol, 63\%) e o diol exo (15) (cristais brancos, 0,027 g, 0,13 mmol, 30\%).

Dados de (11): $\mathrm{T}_{\mathrm{f}}=92-94{ }^{\circ} \mathrm{C}$. IV $\left(\mathrm{KBr}, \mathrm{cm}^{-1}\right) \overline{\mathrm{v}}_{\max }: 3490,3367$, 3227, 2969, 2937, 2869, 1654, 1473, 1458, 1382, 1301, 1199, 1149, $1037,1007,871,836,798,724,651 . \mathbf{R M N}^{\mathbf{1}} \mathbf{H}\left(200 \mathrm{MHz}, \mathrm{CDCl}_{3}\right)$ $\delta$ : $0,97\left(\mathrm{~d}, 3 \mathrm{H}, J_{10,9}=6,5 \mathrm{~Hz}, \mathrm{Me}-10\right), 1,02\left(\mathrm{~d}, 3 \mathrm{H}, J_{11,9}=6,5 \mathrm{~Hz}, \mathrm{Me}-\right.$ 11), 1,33 (s, 3H, Me-12), 1,47-1,72 (m, 2H, H-9 e H-2ß), 1,95-1,97 (m, 2H, H-4 e H-2 $\alpha$ ), 2,55 (s, 1H, OH), 3,05 (s, 1H, OH), 4,09 (d, $\left.1 \mathrm{H}, J_{13 \mathrm{a}, 13 \mathrm{~b}}=12,6 \mathrm{~Hz}, \mathrm{H}-13 \mathrm{a}\right), 4,13(\mathrm{~s}, 1 \mathrm{H}, \mathrm{H}-3), 4,30$ (d, 1H, $J_{13 \mathrm{~b}, 13 \mathrm{a}}=$ $12,6 \mathrm{~Hz}, \mathrm{H}-13 \mathrm{~b}), 4,75$ (s, 1H, H-5), 6,19 (s, 1H, H-6). RMN ${ }^{13} \mathbf{C}(50$ $\left.\mathrm{MHz}, \mathrm{CDCl}_{3}\right) \delta: 20,5^{*}(\mathrm{Me}-10), 21,3 *(\mathrm{Me}-11), 22,1 *(\mathrm{C}-9), 25,6 *$ (Me-12), 41,8 (C-2), 48,7 (C-4), 58,0 (C-13), 66,7 (C-3), 79,3 (C5), 82,6 (C-1), 130,5 (C-6), 148,4 (C-7). EM, m/z (\%): 212(M+, $\left.\mathrm{C}_{12} \mathrm{H}_{20} \mathrm{O}_{3}, 3\right), 194$ (2), 181 (2), 151 (2), 109 (14), 95 (11), 69 (8), 55 (13), 43 (100). *As atribuições podem estar trocadas.

Dados de (15): $\mathrm{T}_{\mathrm{f}}=122-124{ }^{\circ} \mathrm{C} \mathbf{I V}\left(\mathrm{KBr}, \mathrm{cm}^{-1}\right) \overline{\mathrm{v}}_{\max }: 3373,2957$, 2931, 2873, 1654, 1465, 1378, 1312, 1213, 1161, 1033, 969, 857, 805 e 785 . $\mathbf{R M N}^{1} \mathbf{H}\left(200 \mathrm{MHz}, \mathrm{CDCl}_{3}\right) \delta: 0,90\left(\mathrm{~d}, 3 \mathrm{H}, J_{109}=6,9 \mathrm{~Hz}\right.$, Me-10), 1,08 (d, 3H, $\left.J_{11,9}=6,9 \mathrm{~Hz}, \mathrm{Me}-11\right), 1,33$ (s, 3H, Me-12),

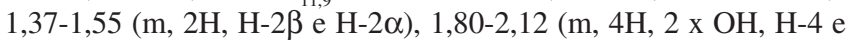
H-9), 3,55-3, 67 (m, 1H, H-3), 4,13 (d, 1H, $\left.J_{13 \mathrm{a}, 13 \mathrm{~b}}=14,7 \mathrm{~Hz}, \mathrm{H}-13 \mathrm{a}\right)$, $4,27\left(\mathrm{~d}, J_{13 \mathrm{~b}, 13 \mathrm{a}}=14,7 \mathrm{~Hz}, \mathrm{H}-13 \mathrm{~b}\right), 4,70(\mathrm{~s}, 1 \mathrm{H}, \mathrm{H}-5)$, e 5,87 (d, 1H, $\left.J_{6,5}=1,7 \mathrm{~Hz}, \mathrm{H}-6\right) . \mathbf{R M N}{ }^{13} \mathbf{C}\left(50 \mathrm{MHz}, \mathrm{CDCl}_{3}\right) \delta: 20,0 *(\mathrm{Me}-10)$, $21,9 *(\mathrm{Me}-11), 22,2 *(\mathrm{C}-9), 26,9 *(\mathrm{Me}-12), 41,5$ (C-2), 51,16 (C4), 58,4 (C-13), 68,2 (C-3), 78,5 (C-5), 83,7 (C-1), 125,2 (C-6) e 
146,8 (C-7). EM, m/z (\%): $212\left(\mathrm{M}^{+}, \mathrm{C}_{12} \mathrm{H}_{20} \mathrm{O}_{3}, 3\right), 194$ (2), 181 (3), 151 (13), 109 (9), 95 (17), 69 (8), 55 (19), 43 (100). *As atribuições podem estar trocadas.

7-formil-4 $\alpha$-isopropil-1 $\alpha$-metil-8-oxabiciclo[3.2.1]oct-6-en-3ona (16) e 7-clorometill-4 $\alpha$-isopropil-1 $\alpha$-metil-8-oxabiciclo[3.2.1] oct-6-en-3-ona (17): A uma mistura de cloreto de oxalila $(0,1 \mathrm{~mL}$, $1,1 \mathrm{mmol})$ em diclorometano seco $(10 \mathrm{~mL})$, sob agitação magnética e $-78{ }^{\circ} \mathrm{C}$, adicionou-se dimetilsulfóxido $(0,15 \mathrm{~mL}, 1,94 \mathrm{mmol})$ diluído em $3 \mathrm{~mL}$ de diclorometano. Após $30 \mathrm{~min}$ a solução do cicloaduto (8) $(0,16 \mathrm{~g}, 0,76 \mathrm{mmol}, 3 \mathrm{~mL}$ de diclorometano) foi adicionada e a mistura mantida sob agitação magnética, a $-78^{\circ} \mathrm{C}$, por $2 \mathrm{~h}$. Em seguida, trietilamina $(0,7 \mathrm{~mL}, 5 \mathrm{mmol})$ foi adicionada, mantendo-se a temperatura de $-78{ }^{\circ} \mathrm{C}$ por $30 \mathrm{~min}$. Após decorrido esse tempo, a reação foi deixada à temperatura ambiente durante $21 \mathrm{~h}$. Em seguida, foram adicionados $15 \mathrm{~mL}$ de água destilada e o produto foi extraído com diclorometano (4 x $15 \mathrm{~mL})$. A fase orgânica foi lavada com solução de ácido clorídrico $1 \%(7 \mathrm{~mL})$, solução aquosa de $\mathrm{NaHCO}_{3}$ $5 \%$ (7 mL), solução saturada de $\mathrm{NaCl}(10 \mathrm{~mL})$, secada sobre $\mathrm{MgSO}_{4}$ e concentrada em evaporador rotatório. Foi obtido um óleo marrom, cuja purificação em coluna de sílica gel (hexano:éter dietílico 5:1) forneceu o aldeído (16) (óleo amarelo, 0,05 g, 0,24 mmol, 32\%) e o clorocicloaduto (17) (óleo amarelo, 0,033 g, 0,15 mmol, 20\%).

Dados de (16): IV (filme líquido, $\mathrm{cm}^{-1}$ ) $\overline{\mathrm{v}}_{\max } 3407,3074,2963$, 2935, 2874, 2735, 1712, 1683, 1607, 1467, 1380, 1327, 1285, 1197, $1169,1053,1025,963,875,850,757,696,635 . \mathbf{R M N}^{\mathbf{1}} \mathbf{H}(200$ $\left.\mathrm{MHz}, \mathrm{CDCl}_{3}\right) \delta: 0,93\left(\mathrm{~d}, 3 \mathrm{H}, J_{10,9}=6,8 \mathrm{~Hz}, \mathrm{Me}-10\right), 1,07(\mathrm{~d}, 3 \mathrm{H}$, $J_{11,9}=6,8 \mathrm{~Hz}, \mathrm{Me}-11$ ), 1,64 (s, 3H, Me-12), 2,01-2,21 (oct., $1 \mathrm{H}, J_{9,11}=$ $\left.J_{9,10}=J_{9,4}=6,8 \mathrm{~Hz}, \mathrm{H}-9\right), 2,49\left(\mathrm{~d}, 1 \mathrm{H}, J_{2 \alpha, 2 \beta}=15,4 \mathrm{~Hz}, \mathrm{H}-2 \alpha\right), 2,55(\mathrm{~d}$, $\left.1 \mathrm{H}, J_{2 \beta, 2 \alpha}=15,4 \mathrm{~Hz}, \mathrm{H}-2 \beta\right), 2,63\left(\mathrm{dd}, 1 \mathrm{H}, J_{4,9}=6,8 \mathrm{~Hz}\right.$ e $J_{4,5}=4,8 \mathrm{~Hz}$, $\mathrm{H}-4), 5,18\left(\mathrm{dd}, 1 \mathrm{H}, J_{5,4}=4,8 \mathrm{~Hz}\right.$ e $\left.J_{5,6}=1,9 \mathrm{~Hz}, \mathrm{H}-5\right), 7,09(\mathrm{~d}, 1 \mathrm{H}$, $\left.J_{6,5}=1,9 \mathrm{~Hz}, \mathrm{H}-6\right)$ e 9,76 (s, $\left.1 \mathrm{H}, \mathrm{H}-13\right)$. RMN ${ }^{13} \mathbf{C}\left(50 \mathrm{MHz}, \mathrm{CDCl}_{3}\right)$ $\delta: 20,2 *$ (Me-10), 21,7* (Me-11), 22,3* (C-9), 24,5* (Me-12), 51,5 (C-2), 61,1 (C-4), 78,8 (C-5), 84,3 (C-1), 149,1 (C-6), 149,6 (C-7), 186,3 (C-13) e 205,4 (C-3). EM m/z (\%):208 ( $\left.\mathbf{M}^{+}, \mathrm{C}_{12} \mathrm{H}_{16} \mathrm{O}_{3}, 3\right), 180$ (3), 179 (19), 151 (10), 137 (32), 109 (21), 83 (14), 69 (95), 55 (16), 43 (100). *As atribuições podem estar trocadas.

Dados de (17): IV (filme líquido, $\mathrm{cm}^{-1}$ ) $\bar{v}_{\text {max }}$ 2962, 2932, 2872, 1710, 1459, 1438, 1381, 1327, 1264, 1167, 1020, 963, 948, 875, 739 e $647 . \mathbf{R M N}^{1} \mathbf{H}\left(200 \mathrm{MHz}, \mathrm{CDCl}_{3}\right) \delta: 0,87\left(\mathrm{~d}, 3 \mathrm{H}, J_{10.9}=6,9 \mathrm{~Hz}\right.$, Me-10), 1,05 (d, 3H, $\left.J_{11,9}=6,9 \mathrm{~Hz}, \mathrm{Me}-11\right), 1,49$ (s, 3H, Me-12), 1,97-2,11 (m, 1H, H-9), 2,43-2,56 (m, 3H, H-2 $\beta$, H-2 $\alpha$ e H-4), 4,07 (d complexo, 2H, H-13a e H-13b), 4,93-4,98 (m, 1H, H-5) e 6,17 (sl, 1H, H-6). RMN ${ }^{13} \mathbf{C}\left(50 \mathrm{MHz}, \mathrm{CDCl}_{3}\right) \delta: 20,0^{*}(\mathrm{Me}-10), 21,5^{*}$ (Me-11), 22,4* (C-9), 24,6* (Me-12), 37,7 (C-13), 52,1 (C-2), 60,8 (C-4), 78,6 (C-1), 84,8 (C-5), 131,3 (C-6), 145,1 (C-7) e 206,2 (C3). $\mathbf{E M ~ m / z ~ ( \% ) : ~} 228\left(\mathrm{M}^{+}, \mathrm{C}_{12} \mathrm{H}_{17} \mathrm{ClO}_{2}, 2\right), 213$ (1), 185 (8), 150 (17), 129 (40), 121 (6), 108 (19), 95 (24), 69 (63), 55 (11), 43 (100). *As atribuições podem estar trocadas.

7-formil-2 $\alpha$-isopropil-1 $\alpha$-metil-8-oxabiciclo[3.2.1]oct-6-en-3ona (18): A uma mistura de cloreto de oxalila $(0,12 \mathrm{~mL}, 1,31 \mathrm{mmol})$ em diclorometano $(10 \mathrm{~mL})$, sob agitação magnética a $-78^{\circ} \mathrm{C}$, adicionou-se dimetilsulfóxido $(0,18 \mathrm{~mL}, 2,3 \mathrm{mmol})$ diluído em $3 \mathrm{~mL}$ de diclorometano seco. Após $30 \mathrm{~min}$, a solução do cicloaduto (14) $(0,19 \mathrm{~g}$, $0,9 \mathrm{mmol}, 3 \mathrm{~mL}$ de diclorometano seco) foi adicionada e a mistura mantida sob agitação magnética, a $-78{ }^{\circ} \mathrm{C}$, por $2 \mathrm{~h}$. Em seguida, trietilamina $(0,83 \mathrm{~mL}, 5,94 \mathrm{mmol})$ foi adicionada, mantendo-se a temperatura de $-78{ }^{\circ} \mathrm{C}$ por $30 \mathrm{~min}$. Após esse tempo, a reação foi deixada à temperatura ambiente durante $21 \mathrm{~h}$. Em seguida, foram adicionados $15 \mathrm{~mL}$ de água destilada e o produto foi extraído com diclorometano (4 x 15 mL). A fase orgânica foi lavada com solução de ácido clorídrico $1 \%$ (7 mL), solução aquosa de $\mathrm{NaHCO}_{3} 5 \%$ (7 mL), solução saturada de $\mathrm{NaCl}(10 \mathrm{~mL})$, secada sobre $\mathrm{MgSO}_{4} \mathrm{e}$ concentrada em evaporador rotatório. Foi obtido um óleo marrom, cuja purificação em coluna de sílica gel (hexano:éter dietílico 3:1) forneceu o aldeído (18) (óleo amarelo, 0,075 g, 0,36 mmol, 40\%). $\mathbf{R M N}^{1} \mathbf{H}\left(200 \mathrm{MHz}, \mathrm{CDCl}_{3}\right) \delta: 0,95\left(\mathrm{~d}, 3 \mathrm{H}, J_{10,9}=6,8 \mathrm{~Hz}, \mathrm{Me}-10\right)$, 1,09 (d, 3H, $J_{11,9}=6,8 \mathrm{~Hz}, \mathrm{Me}-11$ ), 1,30 (s, 3H, Me-12), 2,00-2,40

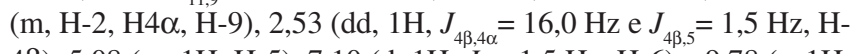
$4 \beta), 5,08$ (m, 1H, H-5), 7,10 (d, 1H, J $\left.{ }_{6.5}=1,5 \mathrm{~Hz}, \mathrm{H}-6\right)$ e $9,78(\mathrm{~s}, 1 \mathrm{H}$, $\mathrm{H}-13)$. RMN ${ }^{13} \mathbf{C}\left(50 \mathrm{MHz}, \mathrm{CDCl}_{3}\right) \delta: 20,8^{*}(\mathrm{Me}-10), 21,4^{*}(\mathrm{Me}-$ 11), 21,6* (C-9), 28,3* (Me-12), 51,5 (C-4), 59,6 (C-2), 78,3 (C-5), 83,9 (C-1), 149,2 (C-6), 150,6 (C-7), 186,3 (C-13) e 207,3 (C-3). *As atribuições podem estar trocadas.

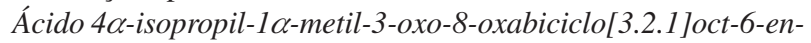
7-óico (19): A uma solução de hidróxido de sódio (0,043g, 1,06 $\mathrm{mmol}$ ) em $3 \mathrm{~mL}$ de água destilada, foi adicionado nitrato de prata $(0,088 \mathrm{~g}, 0,53 \mathrm{mmol})$, sob agitação magnética. A mistura foi resfriada em banho de gelo e, em seguida, adicionou-se a solução do aldeído (16) $(0,05 \mathrm{~g}, 0,24 \mathrm{mmol}) \mathrm{em} 2 \mathrm{~mL}$ de etanol. A reação foi mantida sob agitação magnética, à temperatura ambiente, durante $4 \mathrm{~h}$. Após filtração, o sólido foi lavado com água quente $(10 \mathrm{~mL})$ e, em seguida, com diclorometano $(10 \mathrm{~mL})$. O filtrado foi acidificado com solução de ácido clorídrico $2 \mathrm{~mol} \mathrm{~L}^{-1}$ até $\mathrm{pH} 3$ e a fase aquosa foi extraída com diclorometano (3 x $15 \mathrm{~mL})$. A fase orgânica foi secada com $\mathrm{MgSO}_{4}$ e concentrada em evaporador rotatório. Obteve-se um óleo escuro que, após purificação por cromatografia em coluna de sílica gel (éter dietílico) forneceu o ácido (19) (cristais brancos, 0,042 g, $0,19 \mathrm{mmol}, 78 \%)$, sob a forma de cristais brancos. IV $\left(\mathrm{KBr}, \mathrm{cm}^{-1}\right)$ $\bar{v}_{\max } 3200-2500,2965,2882,1713,1616,1429,1381,1177,1068$, $1021,766,739 . \mathbf{R M N}^{1} \mathbf{H}\left(200 \mathrm{MHz}, \mathrm{CDCl}_{3}\right) \delta: 0,83\left(\mathrm{~d}, 3 \mathrm{H}, J_{10,9}=\right.$ 6,8 Hz, Me-10), 0,90 (d, 3H, $\left.J_{11,9}=6,8 \mathrm{~Hz}, \mathrm{Me}-11\right), 1,43$ (s, 3H, Me12), 2,05-2,70 (m, 4H, H-2 $\beta$, H-2 $\alpha, \mathrm{H}-4, \mathrm{H}-9$ ), 4,75 (d, $1 \mathrm{H}, J_{5,6}=1,9$ $\mathrm{Hz}, \mathrm{H}-5)$ e 7,41 (d, 1H, $\left.J_{6.5}=1,9 \mathrm{~Hz}, \mathrm{H}-6\right), 12,0$ (sl, $\left.1 \mathrm{H}, \mathrm{COOH}\right)$. $\mathbf{E M ~ m / z ~ ( \% ) : ~} 179$ (5, M+-COOH), 152 (21), 151 (10), 126 (21), 110 (86), 97 (40), 84 (16), 69 (52), 55 (25), 43 (100).

\section{Ensaios biológicos}

Soluções dos compostos (8), (11), (15), (16), (17) e (19) foram adicionadas, separadamente, às placas de Petri contendo areia lavada. Em seguida, foram colocadas sementes de sorgo (Sorghum bicolor L.) pré-germinadas por $18 \mathrm{~h}$ à temperatura de $28{ }^{\circ} \mathrm{C}$. As placas foram incubadas à temperatura de $28^{\circ} \mathrm{C}$, sob inclinação de $75^{\circ}$ durante $48 \mathrm{~h}$ quando, então, os comprimentos das radículas foram medidos. As percentagens de inibição das raízes foram calculadas de acordo com as observações do tratamento controle, realizado nas mesmas condições descritas. $\mathrm{O}$ delineamento experimental foi inteiramente casualizado com quatro repetições. Todos os dados observados foram analisados estatisticamente e, para comparação das médias utilizou-se o teste de Tukey a $5 \%$ de probabilidade ${ }^{14}$. O mesmo procedimento foi realizado com sementes de pepino (Cucumis sativus L.). Para a avaliação do efeito dos compostos (8), (11), (15), (16) e (19) sobre o desenvolvimento do sistema radicular e da parte aérea foram utilizados, além de sorgo e pepino, capim-braquiária (B. decumbens Stapf) e desmódio (D. tortuosum (Sw.) DC.), de acordo com metodologia proposta por Parker ${ }^{15}$. Esse experimento foi conduzido em potes plásticos, nos quais adicionou-se uma mistura de areia lavada e solução dos compostos a serem avaliados. As sementes foram semeadas a $1 \mathrm{~cm}$ de profundidade e os potes mantidos em sala de crescimento sob luminosidade e temperatura controladas. Os potes foram irrigados três vezes ao dia com água destilada e três vezes por semana com solução nutritiva comercial. Após 15 dias foi realizada a colheita das plantas. O material coletado foi seco em estufa de ventilação forçada a $75{ }^{\circ} \mathrm{C}$ durante $72 \mathrm{~h}$ e, em seguida, determinou-se a biomassa seca da parte aérea e do sistema radicular, calculando-se a percentagem de inibição para os diferentes tratamentos, em relação ao tratamento controle. 
Para ambos os experimentos os produtos foram avaliados na concentração de 5,5 ppm. As soluções foram preparadas utilizandose xileno, pentan-3-ona, Tween 40 e água destilada, conforme metodologia descrita na literatura ${ }^{16}$.

\section{RESULTADOS E DISCUSSÃO}

\section{Síntese}

O delineamento experimental para a síntese dos análogos oxigenados do ácido helmintospórico fundamentou-se na reação de cicloadição [3+4], que utiliza cátions oxialílicos, gerados in situ a partir de halocetonas e dienos. Essa metodologia gera cicloadutos com grande potencial para modificações químicas ${ }^{12,17}$.

O 3-hidroximetil-2-metilfurano (12) e a 1,1,3,3-tetrabromo-4metilpentan-2-ona (13) foram sintetizados conforme procedimento descrito anteriormente ${ }^{11}$ e obtidos com rendimentos de 95 e $99 \%$, respectivamente. A reação de cicloadição [3+4] entre o dieno (12) e a tetrabromocetona (13) resultou inicialmente em cicloadutos dibromados (não isolados), cuja redução com amálgama de $\mathrm{Zn} / \mathrm{Cu}$, em presença de metanol forneceu os álcoois (8) (37\%, previamente descrito $\left.^{11}\right)$ e (14) (12\%) (Esquema 1). Em trabalho anterior ${ }^{11}$, o composto $(\mathbf{8})$ foi preparado em menor escala $(5 \mathrm{mmol})$, sendo obtido com rendimento de $54 \%$. Essa diferença de rendimento pode ser devida a vários fatores, como qualidade dos reagentes, pureza dos solventes e escala da reação. Portanto, tal diferença mostra que a reação de cicloadição em questão é bastante sensível às condições experimentais. Entretanto, como a reação não foi otimizada, não há

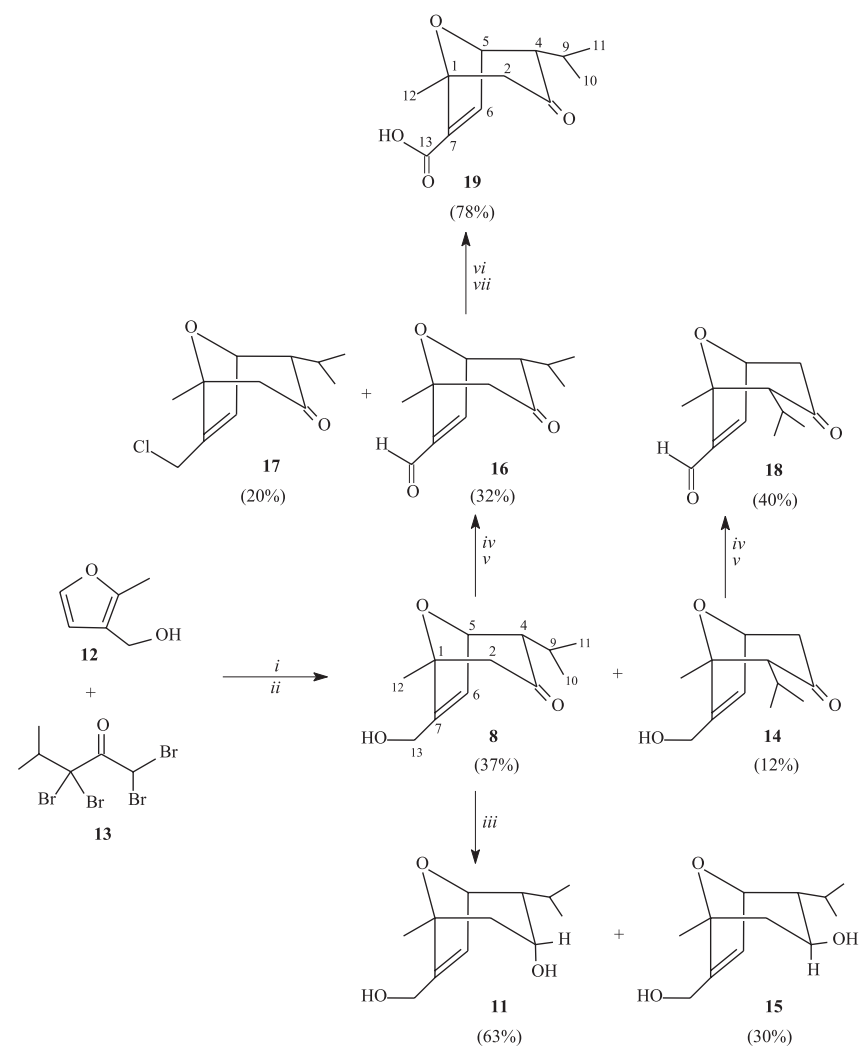

Esquema 1. i) $\mathrm{Et}_{2} \mathrm{Zn}$, Benzeno, $10 \mathrm{~h}, 25^{\circ} \mathrm{C}$; ii) $\mathrm{Zn} / \mathrm{Cu}$; $\mathrm{NH}_{4} \mathrm{Cl}, \mathrm{MeOH}, 6 \mathrm{~h}$, $25^{\circ} \mathrm{C}$; iii) $\mathrm{NaBH}_{4}, \mathrm{EtOH}, 4 \mathrm{~h}, 55^{\circ} \mathrm{C}$; iv) $(\mathrm{COCl})_{2}, \mathrm{DMSO}, \mathrm{CH}_{2} \mathrm{Cl}_{2},-78{ }^{\circ} \mathrm{C}$, $2,5 \mathrm{~h}$; v) $\mathrm{TEA},-78^{\circ} \mathrm{C}, 30 \mathrm{~min}$. $\rightarrow 25^{\circ} \mathrm{C}, 21 \mathrm{~h}$; vi) $\mathrm{NaOH}, \mathrm{AgNO}_{3}, 25^{\circ} \mathrm{C}, 4 \mathrm{~h}$; vii) $\mathrm{HCl}$ como se saber precisamente quais são os fatores experimentais limitantes. A formação dos isômeros (8) e (14) resulta da regiosseletividade da reação de cicloadição [3+4], sendo observada, em ambos os casos, a formação preferencial do isômero com o grupo isopropil em posição equatorial (isômero $\alpha$ ) em relação ao isômero $\beta$ (substituinte em posição axial) ${ }^{12,18}$. A estereoquímica $\alpha$ do grupo isopropil em $\mathrm{C} 4$ no composto $(\mathbf{8})$ foi determinada através da análise do espectro de $\mathrm{RMN}{ }^{1} \mathrm{H}$, que apresentou um duplo dupleto em $\delta$ 2,54 (H4), cujos valores obtidos para as constantes de acoplamento $\left(J_{4,9}=7,3 \mathrm{~Hz}, J_{4,5}=3,0 \mathrm{~Hz}\right)$ estão em concordância com a estrutura proposta. A análise dos espectros no infravermelho, RMN ${ }^{1} \mathrm{H}, \mathrm{RMN}{ }^{13} \mathrm{C}$ e de massas obtidos também foram consistentes com as estruturas propostas para os isômeros (8) e (14).

Para avaliar a influência do grupo carbonila sobre o potencial herbicida dessa classe de compostos, bem como produzir compostos de maior hidrofilicidade, o álcool (8) foi tratado com boroidreto de sódio em metanol ${ }^{11}$. Foram obtidos os isômeros (11) (diol endo, previamente descrito ${ }^{11}$ ) e (15) (diol exo), com rendimentos de 63 e 30\%, respectivamente, a partir de (8). A formação dos dióis endo:exo na proporção 2:1 é decorrente de fatores estruturais que diferenciam o ataque do agente redutor às faces endo e exo da molécula. A fórmula molecular $\mathrm{C}_{12} \mathrm{H}_{20} \mathrm{O}_{3}$ para os dióis (11) e (15) foi confirmada pelo espectro de massas que apresentou um sinal em $\mathrm{m} / \mathrm{z}, 212$ (íon molecular), conforme requerido. A multiplicidade de sinal para o hidrogênio $\mathrm{H} 3$ observada no espectro de $\mathrm{RMN}{ }^{1} \mathrm{H}$ dos isômeros (11) e (15) permitiu a confirmação inequívoca das estruturas propostas. A análise com modelos moleculares mostrou que no caso do diol endo (11), a disposição espacial entre o hidrogênio $\mathrm{H} 3$ e os hidrogênios $\mathrm{H} 2 \alpha, \mathrm{H} 2 \beta$ e H4 ocasiona ângulos diedros de aproximadamente $90^{\circ}$, que resulta em um sinal simples $(\delta 4,13)$ para o hidrogênio H3. Maior complexidade de sinal foi observada para $\mathrm{H} 3$ no caso do diol exo (15) (multipleto, $\delta 3,55-3,67)$, pois neste caso os ângulos diedros observados tornam possíveis os acoplamentos vicinais com os hidrogênios $\mathrm{H} 2 \beta$ e H4.

A introdução do grupo carboxila no esqueleto 8-oxabiciclo foi planejada mediante reações de oxidação (Esquema 1). A metodologia de Swern $^{19}$ foi utilizada para a oxidação do grupo hidroximetil de (8) e (14) que forneceram os aldeídos (16) e (18) com rendimentos de 32 e $40 \%$, respectivamente, a partir dos álcoois de origem. O espectro de massas de (16) mostrou um sinal em $\mathrm{m} / \mathrm{z}, 208$, consistente com a fórmula molecular $\mathrm{C}_{12} \mathrm{H}_{16} \mathrm{O}_{3}$. A carbonila cetônica de (16) foi identificada no espectro no infravermelho pela presença de absorção em $1712 \mathrm{~cm}^{-1}$, e o grupo formil $\alpha, \beta$-insaturado identificado pela absorção em $1683 \mathrm{~cm}^{-1}$. A oxidação do grupo hidroximetil também foi confirmada no espectro de $\mathrm{RMN}{ }^{1} \mathrm{H}$, que apresentou para o hidrogênio aldeídico um sinal simples em $\delta$ 9,76 para (16) e em $\delta$ 9,78 para (18).

A despeito da grande praticidade da metodologia de Swern, o uso de cloreto de oxalila como agente ativante para o dimetilsulfóxido pode levar à formação de produtos secundários decorrentes da cloração eletrofílica ${ }^{20}$. A formação do produto clorado foi observada durante a oxidação do álcool (8), que forneceu o cloro-cicloaduto (17) com rendimento de $20 \%$, a partir de (8). A fórmula molecular $\mathrm{C}_{12} \mathrm{H}_{17} \mathrm{ClO}_{2}$ foi confirmada no espectro de massas pelo sinal do íon molecular em $\mathrm{m} / \mathrm{z}$ 228. A existência de um grupo metileno adicional, em relação ao aldeído (16), foi confirmada no espectro DEPT 135 de (17), que apresentou para o carbono C2 sinal em $\delta 52,1$ e para o carbono alílico C13 sinal em $\delta 37,7$.

Finalmente, o tratamento do aldeído (16) com $\mathrm{AgNO}_{3} / \mathrm{NaOH}$, seguido de acidificação, forneceu o ácido (19) com rendimento de $78 \%^{21}$. O grupo carboxila foi identificado no espectro no infravermelho pela presença da absorção em $1713 \mathrm{~cm}^{-1} \mathrm{e}$ pela banda larga de absorção em 2500-3200 $\mathrm{cm}^{-1}$. 


\section{Ensaios biológicos}

Os ensaios para avaliação da atividade herbicida são geralmente direcionados para dois diferentes tipos de resultados, podendo ser obtidas respostas em partes específicas da planta ou resposta total sobre a mesma. No primeiro caso, por exemplo, pode-se observar inibição ou elongação do caule e/ou das raízes, alterações decorrentes de imperfeições no processo de divisão celular, clorose, necrose e outras variações morfológicas. A resposta total da planta é freqüentemente avaliada através do acúmulo de biomassa seca pela planta, além da sintomatologia de toxicidade. Devem ser selecionadas plantas de diferentes famílias e espécies de fácil germinação e rápido crescimento inicial para se assegurar a confiabilidade de resultados.
Os ensaios foram realizados com os compostos (8), (11), (15), (16), (17) e (19), utilizando-se Sorghum bicolor L., Cucumis sativus L., Brachiaria decumbens Stapf e Desmodium tortuosum (Sw.) DC. Os resultados obtidos estão apresentados nas Tabelas 1 a 3.

O efeito dos compostos sobre o crescimento inicial do sistema radicular foi pouco significativo para S. bicolor (Tabela 1), sendo observadas taxas de inibição menores que $10 \%$. Resultados pouco mais expressivos foram obtidos com C. sativum, observando-se maior efeito de inibição $(27,5 \%)$ para o diol (11) e efeito de indução do crescimento radicular para o álcool (8). Em trabalho anterior ${ }^{11}$, o efeito dos compostos (8) e (11) foi avaliado sobre essas mesmas plantas em um ensaio biológico mais simples (papel de filtro). Foram observados resultados diferentes em relação aos resultados aqui

Tabela 1.- Efeito dos compostos $\mathbf{8 , 1 1}, \mathbf{1 5}, \mathbf{1 6}, \mathbf{1 7}$ e 19, na concentração de 5,5 ppm, sobre o desenvolvimento do sistema radicular de Sorghum bicolor L. e Cucumis sativus L., 48 h após a semeadura

\begin{tabular}{lcccr}
\hline \multirow{2}{*}{ Tratamentos } & \multicolumn{2}{c}{ S. bicolor } & & \multicolumn{2}{c}{ C. sativus } \\
\cline { 2 - 3 } & $\begin{array}{c}\text { Comprimento das } \\
\text { raízes/cm* }\end{array}$ & \% inibição & & $\begin{array}{c}\text { Comprimento das } \\
\text { raízes / cm* }\end{array}$ \\
\hline Controle & $7,15 \mathrm{a}$ & 0,0 & $5,41 \mathrm{ab}$ & 0,0 \\
$\mathbf{8}$ & $6,45 \mathrm{a}$ & 9,8 & $6,06 \mathrm{a}$ & $-12,0$ \\
$\mathbf{1 1}$ & $6,58 \mathrm{a}$ & 8,0 & $3,92 \mathrm{~b}$ & 27,5 \\
$\mathbf{1 5}$ & $6,84 \mathrm{a}$ & 4,3 & $4,40 \mathrm{~b}$ & 18,7 \\
$\mathbf{1 6}$ & $6,56 \mathrm{a}$ & 8,2 & $4,80 \mathrm{ab}$ & 11,3 \\
$\mathbf{1 7}$ & $6,29 \mathrm{a}$ & 1,4 & $4,33 \mathrm{~b}$ & 20,0 \\
$\mathbf{1 9}$ & $6,67 \mathrm{a}$ & 6,7 & $4,43 \mathrm{~b}$ & 18,1 \\
\hline
\end{tabular}

*Médias seguidas por uma mesma letra não diferem entre si pelo Teste de Tukey a 5\% de probabilidade.

Tabela 2. Efeito dos compostos $\mathbf{8}, \mathbf{1 1}, \mathbf{1 5}, \mathbf{1 6}$ e 19, na concentração de 5,5 ppm, sobre o acúmulo de biomassa seca da parte aérea e das raízes de Sorghum bicolor L. e Cucumis sativus L., 15 dias após a semeadura

\begin{tabular}{|c|c|c|c|c|c|c|c|c|}
\hline \multirow{3}{*}{ Tratamentos } & \multicolumn{4}{|c|}{ S. bicolor } & \multicolumn{4}{|c|}{ C. sativus } \\
\hline & \multicolumn{2}{|c|}{ Parte aérea } & \multicolumn{2}{|c|}{ Raiz } & \multicolumn{2}{|c|}{ Parte aérea } & \multicolumn{2}{|c|}{ Raiz } \\
\hline & Massa/g* & \% inibição & Massa/g* & \% inibição & Massa/g* & \% inibição & Massa/g* & \% inibição \\
\hline Controle & $0,068 \mathrm{a}$ & 0,0 & 0,036 a & 0,0 & $0,058 \mathrm{ab}$ & 0,0 & $0,015 \mathrm{ab}$ & 0,0 \\
\hline 8 & $0,053 \mathrm{~b}$ & 22,1 & $0,029 \mathrm{ab}$ & 19,4 & $0,046 \mathrm{ab}$ & 20,7 & $0,014 \mathrm{ab}$ & 6,7 \\
\hline 11 & $0,062 \mathrm{ab}$ & 8,8 & $0,028 \mathrm{ab}$ & 22,2 & $0,053 \mathrm{ab}$ & 8,6 & $0,011 \mathrm{ab}$ & 26,7 \\
\hline 15 & $0,060 \mathrm{ab}$ & 11,8 & $0,025 \mathrm{ab}$ & 30,5 & $0,041 \mathrm{~b}$ & 29,3 & $0,009 \mathrm{~b}$ & 40,0 \\
\hline 16 & $0,062 \mathrm{ab}$ & 8,8 & $0,033 \mathrm{ab}$ & 8,3 & $0,053 \mathrm{ab}$ & 8,6 & $0,011 \mathrm{ab}$ & 26,7 \\
\hline 19 & $0,055 \mathrm{ab}$ & 19,1 & $0,023 \mathrm{ab}$ & 36,1 & $0,056 \mathrm{ab}$ & 3,4 & $0,016 \mathrm{ab}$ & $-6,7$ \\
\hline
\end{tabular}

*Médias seguidas por uma mesma letra não diferem entre si pelo Teste de Tukey a $5 \%$ de probabilidade.

Tabela 3. Efeito dos compostos $\mathbf{8}, \mathbf{1 1}, \mathbf{1 5}, \mathbf{1 6}$ e 19, na concentração de 5,5 ppm, sobre o acúmulo de biomassa seca da parte aérea e raízes de Brachiaria decumbens Stapf. e Desmodium tortuosum (Sw.) DC., 15 dias após a semeadura

\begin{tabular}{llccccccc}
\hline \multirow{2}{*}{ Tratamentos } & \multicolumn{4}{c}{ P. decumbens } & \multicolumn{2}{c}{ P. tortuosum } \\
& Massa/g* & \% inibição & Massa/g* & \% inibição & Massa/g* & \% inibição & Massa/g* & \% inibição \\
\hline Controle & $0,022 \mathrm{a}$ & 0,0 & $0,0097 \mathrm{a}$ & 0,0 & $0,0075 \mathrm{a}$ & 0,0 & $0,0025 \mathrm{a}$ & 0,0 \\
$\mathbf{8}$ & $0,018 \mathrm{~b}$ & 18,2 & $0,0078 \mathrm{ab}$ & 19,6 & $0,0046 \mathrm{~b}$ & 37,8 & $0,0011 \mathrm{bc}$ & 56,0 \\
$\mathbf{1 1}$ & $0,020 \mathrm{a}$ & 0 & $0,0082 \mathrm{ab}$ & 15,5 & $0,0050 \mathrm{~b}$ & 32,4 & $0,0007 \mathrm{bc}$ & 72,0 \\
$\mathbf{1 5}$ & $0,013 \mathrm{~b}$ & 40,9 & $0,0042 \mathrm{~b}$ & 56,7 & $0,0042 \mathrm{~b}$ & 43,2 & $0,0006 \mathrm{c}$ & 76,0 \\
$\mathbf{1 6}$ & $0,019 \mathrm{ab}$ & 13,1 & $0,0087 \mathrm{ab}$ & 10,3 & $0,0040 \mathrm{~b}$ & 45,9 & $0,0008 \mathrm{bc}$ & 68,0 \\
$\mathbf{1 9}$ & $0,023 \mathrm{a}$ & $-4,5$ & $0,0065 \mathrm{ab}$ & 33,0 & $0,0051 \mathrm{~b}$ & 31,1 & $0,0009 \mathrm{bc}$ & 64,0 \\
\hline
\end{tabular}

*Médias seguidas por uma mesma letra não diferem entre si pelo Teste de Tukey a 5\% de probabilidade. 
descritos. Anteriormente, o composto (8), na concentração de 100 ppm, causou $35 \%$ de inibição sobre o crescimento radicular de $S$. bicolor e estímulo de $14 \%$ sobre o desenvolvimento do sitema radicular de $C$. sativum. Nessa mesma concentração, o composto (11) causou $56 \%$ de inibição sobre o crescimento radicular de $S$. bicolor e estímulo de $15 \%$ sobre o desenvolvimento do sistema radicular de C. sativum. Diante dos resultados preliminares alcançados nesse trabalho, buscou-se avaliar, então, a ação dos compostos sobre o desenvolvimento total da planta. Nesse experimento o potencial herbicida foi avaliado em função da percentagem de inibição do sistema radicular e da parte aérea representados pela biomassa seca.

Nos ensaios com $S$. bicolor a maior taxa de inibição da parte aérea $(22,1 \%)$ foi observada para o álcool (8) (Tabela 2). Os demais compostos apresentaram valores inferiores a $12 \%$ de inibição. Ao se analisar os resultados obtidos com a raiz (Tabela 2) notou-se aumento nos índices de inibição, sendo o efeito mais pronunciado para os compostos (15) (30,5\%) e (19) (36,1\%). O diol (15), juntamente com o álcool (8), foram os compostos que apresentaram maior efeito de inibição sobre a parte aérea de $C$. sativum, sendo observadas taxas de inibição de 29,3 e 20,7\%, respectivamente (Tabela 2). Os resultados obtidos com a raiz mostraram efeito máximo de inibição (40\%) para o diol (15) e para o ácido (19) observou-se efeito de indução sobre o crescimento $(6,7 \%)$.

Os compostos (8), (15) e (19) foram os que apresentaram maior ação herbicida sobre $B$. decumbens (Tabela 3). Foi observado sobre a parte aérea inibição de 40,9\% para o diol (15), 18,2\% para o álcool (8) e efeito de indução sobre o crescimento (4,5\%) para o ácido (19). A maior taxa de inibição $(56,7 \%)$ sobre o sistema radicular foi encontrada para o diol (15), seguindo-se o ácido (19) (33\%) e o álcool (8) $(19,6 \%)$.

Quanto à atividade fitotóxica dos derivados helmintospóricos sintetizados sobre $D$. tortuosum observou-se efeito inibitório sobre a parte aérea variando de $31,1 \%$, no caso do ácido (19), a 45,9\% com o aldeído (16). Melhoria acentuada na inibição ocorreu sobre o sistema radicular, onde todos os compostos apresentaram percentuais de inibição superiores a 50\%. A maior inibição (76\%) foi observada para o diol (15). Considerando que D. tortuosum é uma das espécies de plantas daninhas de difícil controle na cultura da soja ${ }^{22}$, e também a importância da soja na economia mundial, esses resultados tornam-se bastante relevantes. A fitotoxicidade desses compostos foi, também, constatada nos ensaios com $C$. sativus e B. decumbens. $\mathrm{O}$ maior efeito foi observado com $B$. decumbens, pois ocorreu redução no porte das plantas, bem como encurtamento e diminuição do número de raízes secundárias. No caso do $C$. sativus foi observado clorose (amarelecimento) das folhas.

\section{CONCLUSÃO}

Os compostos sintetizados foram obtidos a partir da reação de cicloadição [3+4] entre o 3-hidroximetil-2-metilfurano (12) e a 1,1,3,3-tetrabromo-4-metilpentan-2-ona (13), em um total de quatro etapas. Os compostos (8), (11), (15), (16) (17) e (19) apresentaram baixo potencial fitotóxico sobre o crescimento do sistema radicular de $S$. bicolor e de $C$. sativus. Os resultados variaram de indução ao crescimento (12\%), no caso do álcool $(\mathbf{8})$, à inibição $(27,5 \%)$ com o diol (11). Verificou-se, também, que os compostos (8), (11), (15), (16) e (19) causaram inibição no desenvolvimento total das espécies B. decumbens e D. tortuosum, indicando possibilidade para desenvolvimento de um novo herbicida. As maiores taxas de inibição sobre o crescimento (acúmulo de matéria seca) da parte aérea e da raiz foram proporcionados pelo diol (15) com inibições de 76,0 e 43,2\% sobre $D$. tortuosum, e 56,7 e $40,9 \%$ para a $B$. decumbens, respectivamente, para a parte radicular e aérea dessas espécies.

\section{AGRADECIMENTOS}

À Coordenação de Aperfeiçoamento de Pessoal de Nível Superior (CAPES) pela bolsa de estudo (PSS) e ao Conselho Nacional de Desenvolvimento Cientifico e Tecnológico (CNPq) pelas bolsas de pesquisa (A. J. Demuner e L. C. A. Barbosa) e à Fundação de Amparo à Pesquisa de Minas Gerais (FAPEMIG) e ao CNPq pelo apoio financeiro.

\section{REFERÊNCIAS}

1. Copping, L. G.; Crop Protection Agents From Nature, Natural Products and Analogues, SCI: London, 1996, p. 501.

2. Godfrey, C. R. A.; Agrochemicals From Natural Products, $2^{\text {th }}$ ed., Marcel Dekker: New York, 1994, p. 418.

3. Briquet, M.; Vilret, D.; Goblet, P.; Mesa, M.; Eloy, M. C.; J. Bioenerg. Biomemb. 1978, 30, 285.

4. Corey, E. J.; Nozoe, S.; J. Am. Chem. Soc. 1965, 87, 5728.

5. Sakurai, A.; Tamura, S.; Agric. Biol. Chem. 1965, 29, 407.

6. Cutler, H. G.; Crumley, F. G.; Cox, R. H.; Davis, E. E.; Harper, J. L.; Cole, R. J.; Summer, D. R.; J. Agric. Food Chem. 1982, 30, 658.

7. Kim, B. T.; Soh, C. H.; Murofushi, N.; Yoshida, S; Bioorg. Med. Chem. Lett. 1994, 4, 1847.

8. Turner, J. V.; Anderson, B. F.; Mander, L. N.; Aust. J. Chem. 1980, 33, 1061.

9. Mander, L. N.; Prager, R. H.; Turner, J. V.; Aust. J. Chem. 1974, 27, 2645.

10. Barbosa, L. C. A.; Demuner, A. J.; Borges, E. E. L.; Mann, J.; J. Braz. Chem. Soc. 1997, 8, 19.

11. Demuner, A. J.; Barbosa, L. C. A.; Piló-Veloso, D.; J. Agric. Food Chem. 1998, 46, 1173.

12. Demuner, A. J.; Barbosa, L C. A.; Piló-Veloso, D.; Quim. Nova 1997, 20, 18.

13. Perrin, D. D.; Armarego, W. L.; Purification of Laboratory Chemicals, $3^{\text {th }}$ ed., Butterworth-Heinemann Ltd.: Londres, 1988, p. 340.

14. Gomes, F. P.; Curso de Estatística Experimental, $3^{\mathrm{a}}$ ed., Nobel: Piracicaba, 1990, p. 404.

15. Parker, C.; Weed Research 1965, 5, 181.

16. Andreão, A.; Dissertação de Mestrado, Universidade Federal de Viçosa, Brasil, 1998.

17. Barbosa, L. C. A.; Maltha, C. R. A.; Borges, E. E. L.; Quim. Nova 2002, 25, 203.

18. Mann, J.; Holland, J. H.; Tetrahedron 1987, 43, 2533.

19. Mancuso, A. J.; Huang, S. L.; Swern, D.; J. Org. Chem. 1978, 43, 2480.

20. Trost, B. M.; Fleming, I.; Comprehensive Organic Synthesis - Selectivity, Strategy \& Efficiency in Modern Organic Chemistry, $2^{\text {th }}$ ed., Pergamon Press: Oxford, 1993, cap. 2.

21. Baker, W. R.; Coates, R. M.; J. Org. Chem. 1979, 44, 1022.

22. Lorenzi, H.; Manual de Identificação e Controle de Plantas Daninhas, $5^{\mathrm{a}}$ ed., Plantarum: Nova Odessa, 2000, p. 339. 